\title{
A COUPLED-FIELD ANALYSIS ON RF CAVITY
}

\author{
M. C. Lin, Ch. Wang, L. H. Chang, G. H. Luo, P. J. Chou, \\ Synchrotron Radiation Research Center, Science-Based Industrial Park, Hsinchu 300, Taiwan \\ M. J. Huang, \\ Department of Mechanical Engineering, National Taiwan University, Taipei, Taiwan
}

\begin{abstract}
A coupled-field analysis process for a RF cavity structure has been established. The commercial code ANSYS is used as the solver which successfully links analyses of different fields, including electromagnetics, heat transfer, and structure mechanics. The computation efficiency is thus dramatically improved. The calculated RF characteristics of a pill-box cavity match the theoretical predictions pretty well. The following thermal and structural computation based on the surface power loss are also performed. This coupled-field analysis process has been applied to a cavity in design. The computed results associated with RF are compared to the ones calculated by other codes.
\end{abstract}

\section{INTRODUCTION}

For the room-temperature radio-frequency cavities used in the synchrotron radiation facilities, the heat generated by the eddy current induced on the cavity shall be cooled by water. It means not only the electromagnetic characteristics inside the cavity but also the thermal distribution and structural deformation are in need for a design of a RF cavity. Usually several numerical codes are used for the computations of the electromagnetic field, thermal distribution, and structure deformation separately. Thus the time-consuming work of model establishment has to be done at every analysis phase and the rules of each software must be followed individually.

The commercial finite-element-analysis code ANSYS provides the ability to link the thermal and solid analyses with versions earlier than 5.4. For versions after 5.4, ANSYS provides the high-frequency (HF) analysis module and associated elements. This new module was applied to a calculation for PEP-II RF cavity to link with the RF loss and thermal distribution [1, 2], in which the 10-node tetrahedral elements HF119 and SOLID87 were used for electromagnetic and thermal calculations, respectively.

Obviously this coupled-field analysis is more efficient because the model can be established by one single software and related data can be transferred easily in between elements due to same mesh employed. In this work we establish a similar analysis process also under ANSYS. Besides, the commercial code MSC/PATRAN is used as the pre-processor to establish the geometry model and generate ANSYS-compatible mesh. Though MSC/PATRAN is better in generating mapped mesh and in mesh size control, it supports only thermal and structure analyses for ANSYS. Thus a modification on the generated input file is necessary before running at ANSYS HF analysis environment. Because of mapped mesh, the brick elements HF120, SOLID90, and SOLID95 are used for electromagnetic, thermal, and structure calculations, respectively.

\section{TEST WITH A PILL-BOX CAVITY}

A test on a pill-box cavity is performed to check this coupled-field analysis process. Only the fundamental longitudinal accelerating mode $\mathrm{TM}_{010}$ is considered herein.

\subsection{Analytical Predictions}

For a pill-box cavity, its theoretical resonant frequency $f_{0}$ for $\mathrm{TM}_{010}$ mode is predicted as

$$
f_{0}=\frac{c}{2 \pi} \frac{2.405}{R}
$$

in which $\mathrm{c}$ is the light speed and $R$ the cavity radius. For a $500 \mathrm{MHz}$ cavity, its radius is calculated as $0.22966 \mathrm{~m}$. The cavity length $L$ is chosen as $0.05 \mathrm{~m}$ in this test case, while the wavelength of a $500 \mathrm{MHz}$ cavity is $0.6 \mathrm{~m}$. The unloaded quality factor $Q_{0}$ of such a cavity is

$$
Q_{0}=\eta \sigma \delta \frac{2.405}{2}\left(\frac{R}{L}+1\right)^{-1}
$$

in which $\eta, \sigma$, and $\delta$ are the intrinsic impedance, conductivity, and skin depth of the cavity wall material. The theoretical $Q_{0}$ is 13896.31 for the test copper cavity.

\subsection{Computed Results}

Because of symmetry, one quarter of the pill-box cavity is modeled. In the HF calculation phase, the element numbers along the radius and circumference directions are defined as $\mathrm{n} 1$ and $\mathrm{n} 2$, respectively, while the longitudinal element number $\mathrm{n} 3$ keeps 5 always. The computed resonance frequency $f$ is directly given by ANSYS, while the stored electrical energy $\mathrm{E}_{\mathrm{E}}$, stored magnetic energy $\mathrm{E}_{\mathrm{H}}$, and surface power loss $P_{C}$ are calculated by programmed macros in the ANSYS program. The average stored energy is defined as $E_{\text {avg }}=$ $\left(\mathrm{E}_{\mathrm{E}}+\mathrm{E}_{\mathrm{H}}\right) / 2$, and the energy difference as $\Delta \mathrm{E}=\mathrm{E}_{\mathrm{E}}-\mathrm{E}_{\mathrm{H}}$. The quality factor $Q$ is then calculated by

$$
Q=2 \pi f E_{\text {avg }} / P_{c}
$$

The calculated $f$ and $Q$ with different element numbers are compared with the analytical ones as shown in Fig. 1 and Fig. 2. In these two figures, $\Delta \mathrm{f}=f-f_{0}, \Delta \mathrm{Q}=Q-Q_{0}$. The computed energy difference $\Delta \mathrm{E}$, which should be 0 in theory, is also shown and normalized by $\mathrm{E}_{\text {avg. }}$. It is shown 
that the calculated resonance frequency $f$ approaches to the theoretical value $f_{0}$ with an error less than $0.01 \%$ even at a coarse mesh. But the calculated quality factor can have an error less than $0.1 \%$ only at fine mesh, say $n 1=20$ and $n 2=30$. Because $Q$ is related to the stored energy and surface power loss, it means a fine-mesh model is necessary when the surface power loss is concerned. On the other hand, a model with coarse mesh can give a good estimation of resonance frequency, which is beneficial in the initial design for the cavity shape.

Another useful information shown in these two figures is the computed electrical energy increases more than magnetic energy when the radial element number $\mathrm{n} 2$ increases, while magnetic energy increases more when circumferential element number $\mathrm{n} 1$ increases. This should relate to the electrical and magnetic field distribution. However, it could be used to examine the element mesh.

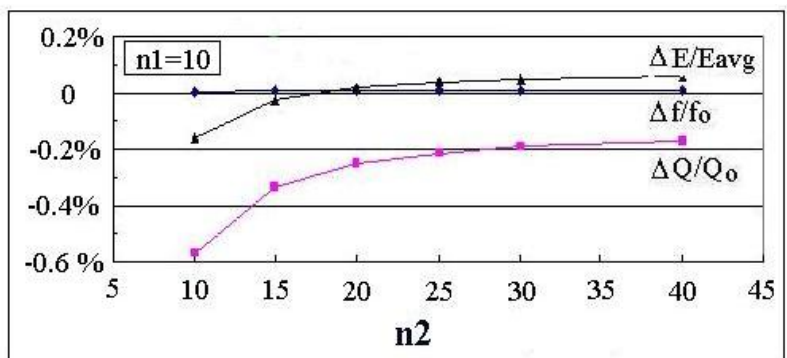

Figure 1: Convergence of computed HF characteristics for a $500 \mathrm{MHz}$ pill-box cavity with increasing radial element number $\mathrm{n} 2$.

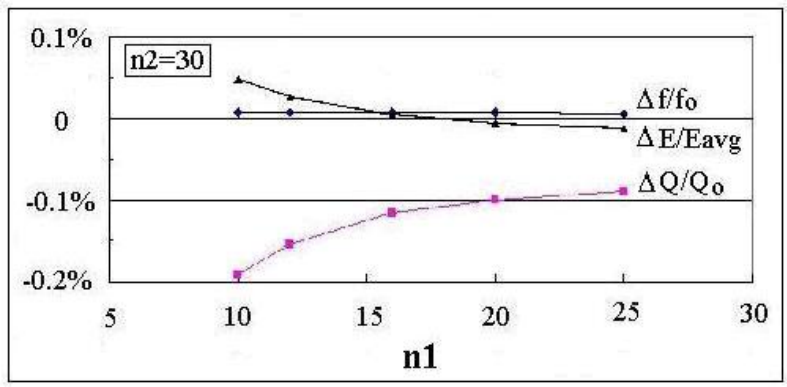

Figure 2: Convergence of computed HF characteristics for a $500 \mathrm{MHz}$ pill-box cavity with increasing circumferential element number $\mathrm{n} 1$.

The surface power loss was then scaled to the desired value by a programmed ANSYS macro. Here it is scaled to an equivalent value of integral electrical voltage 100 $\mathrm{kV}$ along the cavity axis. The peak power density at the surface is $2.78 \mathrm{~W} / \mathrm{cm}^{2}$. A 2 -cm thickness cavity wall was generated for the following thermal analysis. Now the element SOLID90 is used and the wall is divided to 3 element layers with material constants given as copper. Giving a simplified temperature boundary condition of uniform temperature distribution at the cavity outer surface, the distribution of temperature rise can then be computed. The maximum temperature rise is only $1.36{ }^{\circ} \mathrm{C}$ because of the thin wall and good thermal conductivity.
With the temperature distribution as body load and fixed-point boundary condition at "left" cavity center point as well as the symmetric conditions, the structure deformation and thermal stresses are then computed by the structure analysis. Here the SOLID90 elements are transformed to SOLID95 elements. Shown in Fig. 3 are the longitudinal displacement $\mathrm{U}_{\mathrm{Z}}$ and radial displacement $\mathrm{U}_{\mathrm{R}}$ at the cavity outer surface of "right" side. The increasing of $U_{R}$ shows the cavity expands at radius direction, while the decreasing of $U_{z}$ shows the cavity bulging out at the center part. Shown in Fig. 4 is the effective thermal stress. The effective stress is calculated from the normal and shear stresses. It is commonly used to judge whether the material is stretched to plastic range. In this case the peak effective stress is $1.85 \mathrm{~N} / \mathrm{mm}^{2}$ (MPa), far below copper's yielding stress $55 \mathrm{MPa}$.

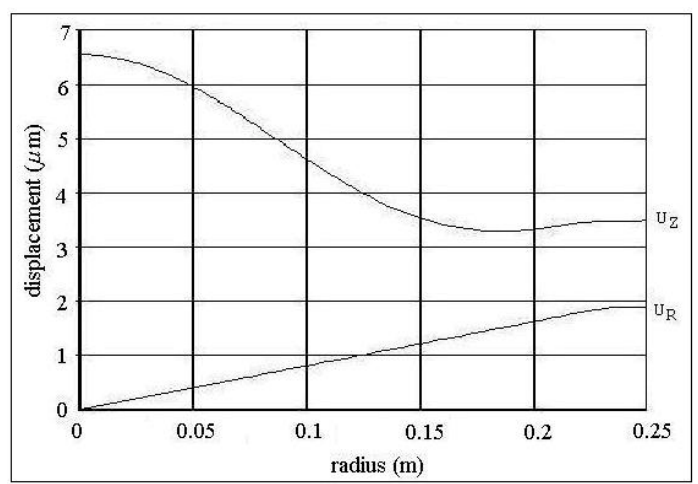

Figure 3: Computed longitudinal and radial displacements $U_{Z}$ and $U_{R}$ at the cavity outer wall of free side, with an integrated longitudinal electrical voltage of $100 \mathrm{kV}$ and uniform temperature distribution at the outer surface.

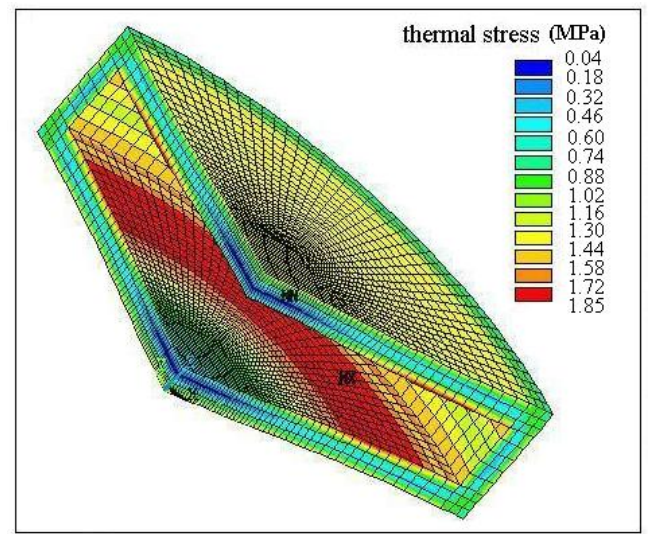

Figure 4: Computed effective stress on the cavity with an integrated longitudinal electrical voltage of $100 \mathrm{kV}$ and uniform temperature distribution at the outer surface.

\section{APPLICATION AND COMPARISON WITH OTHER CODES}

This computation process has been applied to a cavity in design, BESSY II Quasic-HOM free cavity. This cavity shape has been optimized with SEAFISH and URMEL by Weng [3]. The optimized dimensions are adopted here to build the ANSYS analytical model. Because of symmetry, the model is built with one half in 
length and $1 / 6$ in circumference. The computed RF characteristics are listed in Table 1 with the results scaled to $50 \mathrm{~kW}$ surface power loss. Weng's calculating results with SEAFISH and URMEL are also listed for comparison. It is clearly shown that the ANSYS results are close to the others.

Table 1: Computed RF characteristics of a cavity in design at BESSY II.

\begin{tabular}{|c|c|c|c|}
\hline & SEAFISH $^{[3]}$ & URMEL $^{[3]}$ & ANSYS \\
\hline frequency $(\mathrm{MHz})$ & 499.727 & 499.675 & 499.972 \\
\hline Rs, eff (M $(\mathrm{M})$ & 4.95 & 4.86 & 4.96 \\
\hline $\mathrm{Q}$ & 38633.3 & 37972 & 38569 \\
\hline Rs,eff / Q $(\Omega)$ & 128.123 & 128.089 & 128.464 \\
\hline $\mathrm{E}_{\mathrm{p}} @ 50 \mathrm{~kW}(\mathrm{MV} / \mathrm{m})$ & 10.055 & 9.727 & 9.94 \\
\hline $\mathrm{B}_{\mathrm{p}} @ 50 \mathrm{~kW}$ (Gauss) & 92.4 & 91.3 & 92.6 \\
\hline $\mathrm{P} @ 050 \mathrm{~kW}\left(\mathrm{~W} / \mathrm{cm}^{2}\right)$ & 15.4 & 15.1 & 15.8 \\
\hline hot spot $[\mathrm{R}, \mathrm{Z}](\mathrm{mm})$ & {$[72.6,140.1]$} & {$[73.3,141.6]$} & {$[73.1,142.3]$} \\
\hline $\mathrm{Vg} @ 50 \mathrm{~kW}(\mathrm{kV})$ & 703.549 & 697.418 & 703.9 \\
\hline transit-time factor & 0.732 & 0.733 & 0.7322 \\
\hline
\end{tabular}

The accelerating electrical field along the cavity axis is shown in Fig. 5 as scaled to $50 \mathrm{~kW}$ surface power loss. The nose cone tip is at $\mathrm{Z}=0.106 \mathrm{~m}$ while the cavity center is at $\mathrm{Z}=0$, so it is clear that the on-axis accelerating field $E_{z}$ has a peak value of $4.14 \mathrm{MV} / \mathrm{m}$ in between the nose cone tip and cavity center. Besides $E_{z}$ beyond the nose cone can not be neglected. Note that the peak electric field $\mathrm{E}_{\mathrm{p}}, 9.84 \mathrm{MV} / \mathrm{m}$ as listed in Table 1, happens at nose cone tip and is larger than twice of the peak value of on-axis accelerating field.

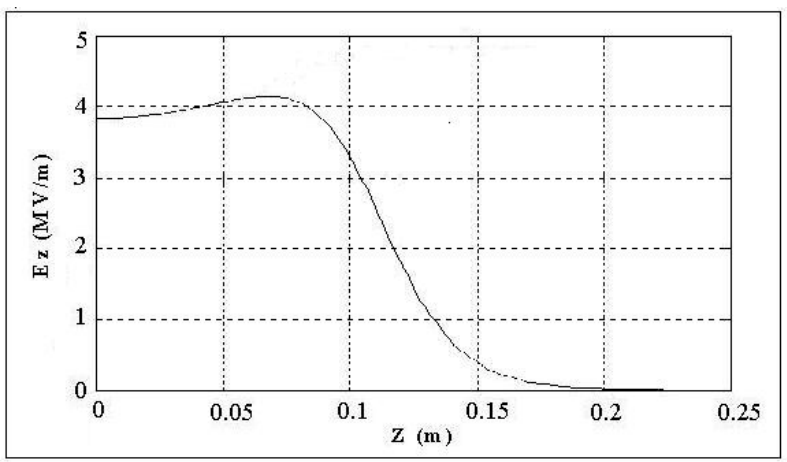

Figure 5: Computed longitudinal electrical field $\mathrm{E}_{\mathrm{z}}$ along cavity axis as scaled to $50 \mathrm{~kW}$ surface power loss for the cavity in design at BESSY II.

The surface loss distribution is shown in Fig. 6 for total $50 \mathrm{~kW}$. The area near the conjunction corner of the nose cone and the vertical wall is most heated. The peak surface loss is $15.83 \mathrm{~kW} / \mathrm{cm}^{2}$. As listed in Table 1 , the axis coordinate $\mathrm{Z}$ of this point is $142.3 \mathrm{~mm}$ while the nose cone tip is at $\mathrm{Z}=106 \mathrm{~mm}$. This provides useful information for following cooling channel layout. There should be more cooling channels in the hot regime to provide better cooling thus the thermal stress can be reduced. The openings for coupler, tuner, and high order mode absorbers are going to be modeled in the near feature. This would take a much larger and more complicated model. The cooling channel layout for actual cavity structure can thus be figured out. Also this computing process can be used to examine and adjust the cooling channel layout before cacity fabrication.

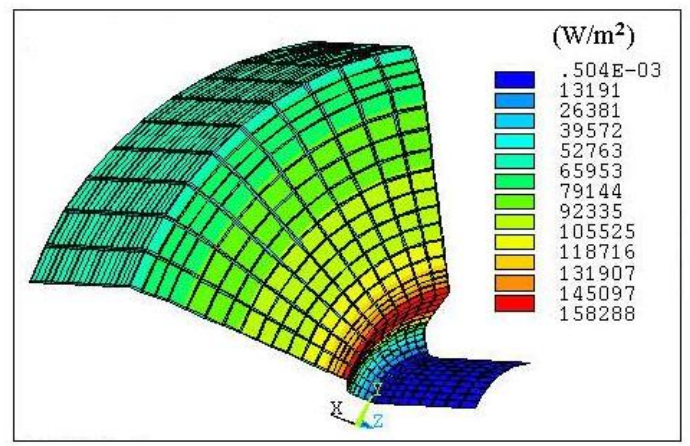

Figure 6: Computed surface loss distribution on the cavity in design at BESSY II as scaled to $50 \mathrm{~kW}$ power loss.

\section{CONCLUSIONS}

A coupled-field analysis process has been established for the cavity design and calculation. The computing is thus simplified and efficiency improved. A test with the pill-box cavity reveals the computing results would approach theoretical predictions with fine mesh. This process is currently being applied to an cavity in design. It is expected that a good cooling channel layout can thus be obtained.

\section{ACKNOWLEDGEMENT}

This work is supported in part by National Science Council of Taiwan under Contract NSC-89-2213-E-213001, and by the National Center For High-Performance Computing for running ANSYS. The authors would like to thank Mr. Neal Hartman (SLAC) for valuable technical discussion.

\section{REFERENCES}

[1] R. A. Rimmer et al., "PEP-II RF Cavity Revisited," LBNL Report No. LBNL-45136, SLAC Report No. LCC-0032, 1999.

[2] R. A. Rimmer et al., "RF Cavity R\&D at LBNL for the NLC Damping Rings, FY1999," SLAC Report No. LCC-0033, 1999.

[3] W. T. Weng, "Shunt impedance of A RF cavity," Master Thesis, Department of Physics, National Tsing Hua University, Taiwan, 2000 (in Chinese). 